\title{
Event-Driven Rules: Experiences in CEP
}

\author{
Paul Vincent \\ TIBCO Software, London, UK \\ pvincentatibco.com
}

\begin{abstract}
Event Driven Architectures (EDA) and Complex Event Processing (CEP) are demonstrating interesting alternatives to the app-server-executingbusiness-logic approaches that are used in both business and cloud IT deployments today. Applying rules (and rule-based inferences) to events is a natural solution for business event processing, and the benefits of the "event-decisionaction" pattern enabled by rules have proved very useful in a number of application cases. Here we introduce how rule-driven CEP is becoming a leading application area for rule technology together with some recent case studies on how declarative rules provide a suitable knowledge representation for eventdriven processes in business applications.
\end{abstract}

